\title{
Correction: Organization of the social cognition network predicts future depression and interpersonal impairment: a prospective family-based study
}

Eyal Abraham, Yun Wang, Connie Svob, David Semanek, Marc J. Gameroff, Stewart A. Shankman (D), Myrna M. Weissman, Ardesheer Talati and Jonathan Posner

(C) The Author(s), under exclusive licence to American College of Neuropsychopharmacology 2021

Neuropsychopharmacology (2022) 47:612; https://doi.org/10.1038/s41386-021-01105-3

Correction to: Neuropsychopharmacology; https://doi.org/10.1038/ s41386-021-01065-8, published online 23 June 2021

In this article, the affiliation from Connie Svob 1,2 was removed during the proofing stage.

1 Department of Psychiatry, Columbia University Vagelos College of Physicians and Surgeons, New York, NY, USA.

2 Divisions of Translational Epidemiology and New York State Psychiatric Institute, New York, NY, USA.
3 Child Psychiatry, New York State Psychiatric Institute, New York, NY, USA.

4 Department of Psychiatry and Behavioral Sciences, Northwestern University, Evanston, USA.

5 Department of Epidemiology, Mailman School of Public Health, Columbia University, New York, NY, USA.

The original article has been corrected. 